\title{
TUBERCULAR LYMPHADENITIS IN PAEDIATRIC PATIENT IN E.N.T. PRACTICE
}

\section{Lateef Chesti ${ }^{1}$, Irfan Iqbal'2, Showkat A. Showkat ${ }^{3}$, Sajad M. Qazi², Ayaz Rehman², Zarka Amin ${ }^{3}$}

\author{
${ }^{1}$ Professor and Head \\ ${ }^{2}$ Associate Professor \\ ${ }^{3}$ Registrar \\ 2Postgraduate Scholar \\ Department of ENT, Head \& Neck Surgery \\ Government Medical College \\ Srinagar - 190 010, India
}

\section{INTRODUCTION}

Tuberculosis is one of the biggest health challenge, the world is facing. Cervical lymphadenitis is a common manifestation of mycobacterial infections encountered in otorhinolaryngologic practices. It may be the manifestation of a systemic tuberculous disease or a unique clinical entity localized to neck. It remains a diagnostic and therapeutic challenge because it minimizes other pathological processes and eyelids in consistent physical and lab findings. A high index of suspicion is needed for the diagnosis of mycobact cervical lymphadenitis and should be considered in the differential diagnosis of a cervical mass especially in endemic areas.

Keywords: Cervicofacial masses, lymphadenopathy FNAB, HPE, developmental malformations, cystic lesions.

\section{MATERIALS \& METHODS}

The present study was conducted in 175 patients with cervicofascial masses who attended the Department of

\section{Correspondence to:}

Dr. Irfan Iqbal

Postgraduate Scholar, Department of Otorhinolaryngology, Government Medical College, Srinagar - 190010, Kashmir (India)

Post Box No. 120 GPO

Email: drirfan2007@gmail.com

Post Box No. 120 GPO

Mobile: 9419038217
Otorhinolaryngology, Head \& Neck Surgery, SMHS Hospital, Government Medical College Srinagar. Both male and female patients below and upto the age of 12 years with cervicofascial mass were taken up for the study. The patients were followed up for a period of six months to one year.

A detailed history regarding presenting symptoms and duration of illness was taken and recorded on a proforma. History included the mode of onset, progression of disease, associated symptoms like pain, 
fewer, discharge, weakness, sore throat, weight loss, any interference with normal functions. Any significant past history was noted. Family, personal and drug history were also noted.

A complete general physical examination and systemic examination was done to exclude any concomitant disease and contraindication for surgical intervention if needed.

A thorough otorhinolaryngological examination was done in each case. A detailed local examination of the mass was performed in a systematic order. Preliminary laboratory investigations, blood chemistry, $x$-ray chest was done.

FNAB was carried out in all cases of the series. Treatment included wait and watch, antibiotic therapy, ATT and surgical intervention. HPE was done in all cases where excision was performed. Postoperative patients were follow up for a period of 6 months and 1 year.

\section{RESULTS}

The present study was carried out on 175 patients below and upto the age of 12 years who presented with cervicofascial masses in the Department of Otorhinolaryngology and Head \& Neck Surgery of Government Medical College Srinagar.

It was found that there were 23 patients (11.5\%) of tubercular lymphadenitis, out of which 7 were males and 16 females in the ratio of 1:2.3
Out of these 23 cases 11 cases (48.5\%) presented with right sided lesion, 4 patients $(17 \%)$ left sided lesion, 6 patients $(26 \%)$ with bilateral lesions and 2 patients (8.5\%) with submental lesion.

The various sites for tubercular lymphadenitis were submandibular (13 out of 23), submental (2 out of 23), posttriangular of neck (5 out of 23 and as multiple neck swelling (2 out of 23).

Constitutional symptoms were not presented in most of the patients. The patients were investigated with routine baseline investigations. ESR was found elevated in $13 \%$ of patients having tubercular lymphadenitis. FNAB yielded a positive diagnosis in 21 out of 23 of the 200 cases of the series. But FNAB was false positive in $12.6 \%$ of patients. Sensitivity of FNAB was found to be $87.4 \%$, two patients diagnosed on FNAB as reactive hyperplasia of lymphnode proved to be tubercular lymphadenitis on HPE.

All patients were put on anti tubercular drug for a period of 9 months. No relapse occurred and no further surgery was required.

\section{DISCUSSION}

Cervical lymphadenopathy is the most common head and neck manifestation of TB and can occur in any age group. The incidence of cervical lymphadenitis has increased in parallel with the increase in the incidence of mycobacterial infection world wide. 
Mycobacterial cervical lymphadenitis is caused either by tuberculous or nontuberculous mycobacteria.

Most series suggest only $10-20 \%$ to have associated pulse disease or to have had a history of contact with TB. Upto one half of these patients will have systemic symptoms. It has been suggested that the bacillus enter via the tonsils as tonsillectomy specimens will often show evidence of TB infection in these patients.

It may present as single or multiple painless lump, mostly located in post cervical or supraclavicular region. The fistula formation may be seen in almost $10 \%$ of the mycobacterium cervical lymphadenitis.

It is important to distinguish between tuberculous and non-tuberculous mycobacterial cervical lymphadenitis because their treatment protocols are different.

The diagnostic needs are high index of suspicion and application of a variety of diagnostic modalities. It should be strongly considered in patients living in endemic areas and immunocompromized people. It is not feasible and practical to apply all the diagnostic procedures to all patients. This would be time counseling and expensive. The test battery should be individualized.

A thorough history and physical examination, tuberculin test, staining for
AFB, radiological examination and FNAC with the help to arrive at an early diagnosis of mycobacterial cervical lymphadenitis will allow early institution of treatment before a final diagnosis can be made by biopsy and culture.

The sensitivity and specificity of FNA cytology in the diagnosis of tubercular lymphadenitis are 88 to $96 \%$ respectively. Combination of FNAB and montoux test increases the diagnostic yield in mycobacterial cervical lymphadenitis.

TB lymphadenitis responds well to ATT and surgery has a limited role in treatment. A surgical intervention in tubercular adenitis should include FNA, drainage and incisional and excisional biopsy.

Anti tubercular drugs include two groups of drugs. First line drug are INH, RMB, ENB, PZA and streptomycin. Second line drug which are less efficacious and more toxic than the first line drugs are caprecomycin, ethionamide, thiacetazone, PAS and cyclosein.

In non-tubercular adenitis, surgery is the treatment of choice. Surgical techniques include aspiration, excision and drainage, cartilage, complete surgical examination of affected lymphnode and overlying skin and selective nodal and functional neck dissection when required. 
Pediatric cervicofascial mass

No response to antibiotics $\geq 2$ meters

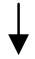

High index of suspicion for TB

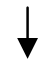

FNAB

$\downarrow$

Cytologic examin stain and culture

$\downarrow$

Granulomatous inflammation

$\downarrow$

(AFB stain)

$\downarrow$

Chest X-ray

TB

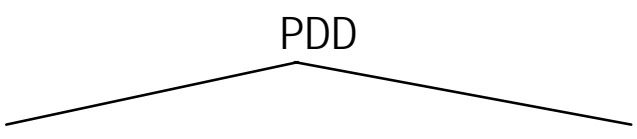

Non TB mycobacterial infection

Medical Management

Surgical Management

\section{REFERENCES}

1. Munck K, Mandpe AH. Mycobacterial infections of the head and neck. Otolaryngol Clin North Am 2003; 36: 569-576.

2. Kanlikama M, Mumbuc S, Bayazit $Y$, Sirikci A. Management strategy of mycobactrial cervical lymphadenitis. J Laryngol Otol 2000; 114: 274-278.

3. Alleva M, Guida RA, Tomo T $3^{\text {rd }}$, Kimmelman CP. Mycobacterial cervical lymphadenitis: A persistent diagnostic problem. Laryngoscope 1988; 98: 855-857. 
4. Penfold DN, Revington PJ. A review of 23 patients with tuberculosis of head and neck. Br J Oral Maxillofac Surg 1996; 34: 508-510.

5. Ibekwe AO, al Shareef Z, al Kindy S. Diagnostic problems of tuberculous cervical adenitis (scrofula). Am J Otolaryngol 1997; 18: 202-205.

6. Chao SS, Loh KS, Tan KK, Chong SM. Tuberculous and nontuberculous cervical lymphadenitis: A clinical rev iew. Otolaryngol Head Neck Surg 2002; 126: 176-179.

7. Tunkel DE. Surgery for cervicofacial nontuberculous mycobactrial adenitis in children: An update. Arch Otolaryngol Head Neck Surg 1999; 125: 1109-1113.

8. Weiler Z, Nelly P, Baruchin AM, Oren S. Diagnosis and treatment of cervical tuberculous lymphadenitis. J Oral Maxillofac Surg 2000; 58: 477-481.

9. Gupta SK, Chugh TD, Sheikh ZA, al-Rubah NA. Cytodiagnosis of tuberculous lympyhadenitis. A correlative study with microbiologic examination. Acta Cytol 1993; 37: 329-332.

10. Lau SK, Wei WI, Hsu C, Engzell UC. Fine needle aspiration biopsy of tuberculous cervical lymphadenopathy. Aust N Z J Surg 1988; 58: 947-950.

11. Russel J. Ord \& Gregory J Matz. Tuberculous cervical lymphadenitis. Arch Otolaryngol May 1974; 99: 327-329. 